\section{$\underset{\substack{\text { hommes } \\ \text { \& migrations }}}{ }$}

\section{Hommes \& migrations}

Revue française de référence sur les dynamiques

migratoires

$1314 \mid 2016$

Migrations chinoises et générations

\title{
Migrer ou rester au village
}

Une génération d'enfants dans l'ombre du miracle économique chinois

\section{Tao Li}

Traducteur : Stéphane Lévêque

\section{(2) OpenEdition \\ Journals}

\section{Édition électronique}

URL : http://journals.openedition.org/hommesmigrations/3627

DOI : 10.4000/hommesmigrations.3627

ISSN : 2262-3353

\section{Éditeur}

Musée national de l'histoire de l'immigration

\section{Édition imprimée}

Date de publication : 1 avril 2016

Pagination : 27-33

ISBN : 978-2-919040-35-3

ISSN : $1142-852 X$

\section{Référence électronique}

Tao Li, « Migrer ou rester au village », Hommes \& migrations [En ligne], 1314 | 2016, mis en ligne le 01 avril 2019, consulté le 20 avril 2019. URL : http://journals.openedition.org/hommesmigrations/3627 ; DOI : 10.4000/hommesmigrations.3627 


\title{
MIGRER OU RESTER AU VILLAGE
}

\section{UNE GÉNÉRATION D'ENFANTS DANS L'OMBRE DU MIRACLE ÉCONOMIQUE CHINOIS}

par TAO LI, post-doctorant à l'Académie chinoise des sciences sociales (Pékin).

\author{
En Chine, I'urbanisation accélérée des années 2000 a entraîné \\ I'augmentation des migrations internes des populations rurales \\ chinoises vers les villes. Des millions d'enfants accompagnent \\ leurs parents dans les grandes villes et plus encore restent \\ dans leur village d'origine. Dans les deux cas, la question de leur \\ scolarisation s'avère préoccupante. Si les villes moyennes \\ en plein développement représentent aujourd'hui un compromis \\ acceptable - en terme de niveau d'éducation et de proximité \\ de la famille -, les écoles restent des lieux de reproduction \\ sociale et participent à la fabrication des inégalités.
}

Depuis le lancement de la politique de réforme et d'ouverture en 1978, les mouvements migratoires internes à la Chine sont massifs entre les zones rurales et les zones urbaines. Au cœur de cet exode rural se trouvent les enfants qui accompagnent leurs parents dans la migration ou qui restent en arrière dans les villages. La migration ou non des enfants pose dans tous les cas le problème de l'enjeu éducatif, alors que dans les grandes villes ils sont souvent exclus des établissements publics et que dans les campagnes les écoles ferment ou ne proposent pas d'éducation de qualité. Avant de décrire ces enjeux et la reproduction des inégalités via la scolarisation, nous rappellerons rapidement les grandes phases d'urbanisation de la Chine et l'impact sur les enfants de migrants internes.

\section{Trois grandes phases d'urbanisation et de migrations internes}

La première phase de migrations internes s'étend des guerres de l'Opium (1848 et 1860), qui ont obligé la Chine à s'ouvrir sur l'étranger, à la fondation de la République populaire de Chine (RPC) en 1949. L'ouverture des villes portuaires et le développement du commerce ont permis l'entrée de capitaux et de technologies industrielles qui ont initié le processus d'urbanisation en Chine. Le Mouvement d'auto-renforcement (1861) a fournitle cadre de l'industrialisation et de l'urbanisation 
de plusieurs villes qui se sont modernisées et ont attiré une main-d'œuvre paysanne de plus en plus nombreuse. En à peine plus d'un siècle - de 1848 à 1949 -, la population urbaine est passée de 20,7 millions à 57,7 millions d'individus et le taux d'urbanisation de $5,1 \%$ à 10,6 \% ${ }^{1}$. Bien que le taux d'urbanisation de 1949 reste inférieur à celui des pays occidentaux - il correspond

La plupart des 270 millions de personnes qui composent

la population migrante

chinoise sont de jeunes

travailleurs. Certains emmènent avec eux leurs enfants pour les scolariser en ville, d'autre pas. à celui de l'Angleterre de 1850 -, l'avancée de l'urbanisation est relativement rapide si l'on tient compte des guerres, des soubresauts politiques, des mouvements de masse, des révolutions et des désastres que la Chine a connus. L'essentiel de la population urbaine se concentre à Shanghai - avec ses 3,48 millions d'habitants en 1936 - et dans une dizaine de grandes villes comptant plus de 500000 habitants, dont des villes côtières où les concessions étrangères ont grandement contribué à cette urbanisation. Sur l'ensemble de la Chine, la répartition de la population demeure fort inégale.

La deuxième phase couvre les trois décennies qui suivent la fondation de la République populaire de Chine (1949-1978). Dans une économie planifiée hautement centralisée et favorisant le développement de l'industrie lourde, le pourcentage de la population urbaine a rapidement augmenté. Afin de réguler les flux de migrants que les villes étaient dans l'incapacité d'absorber, les autorités ont adopté un système de hukou (permis de résidence) qui distinguait les résidents des zones rurales de ceux des zones urbaines et limitait la mobilité vers les villes des premiers. En outre, pendant les dix longues années de la Révolution culturelle (1966-1976), alors que l'économie stagnait, les flux migratoires se sont inversés : les "jeunes instruits", les cadres, les ouvriers et les employés de plus de 3000 villes furent envoyés travailler dans les campagnes. L'urbanisation chinoise a donc connu au cours des trente premières années de la RPC plusieurs étapes : d'abord une progression régulière, puis un recul et enfin une stagnation. Le taux d'urbanisation en 1960 a connu un maximum de 19,75\%, puis est retombé à 17,55 $\%$ en 1977. Mais si l'urbanisation avait suivi la même progression à un taux moyen de 0,59 \% par an comme entre 1949 et 1958 (début du Grand Bond en avant), selon les estimations de l'auteur, on peut considérer que le recul du taux d'urbanisation entre 1960 et 1977 n'est pas de 2 mais de 10 points.

La troisième phase va de 1978 à nos jours, quatre décennies marquées par une politique de réformes économiques et d'ouverture sur le monde. La mise en place d'une économie socialiste de marché a entraîné une accélération du rythme de l'urbanisation chinoise. Trois sous-étapes se dessinent : une reprise de l'urbanisation entre 1978-1984, une stabilisation entre 1985 et 1991 puis un développement rapide de 1992 à nos jours. Si l'on considère le taux d'urbanisation par décennie, on constate une augmentation annuelle moyenne de 0,7 \% entre 1980 et 1990, de $1 \%$ entre 1990 et 2000, de 1,36\% entre 2000 et 2010 et enfin de 1,28\% entre 2010 et 2015. En moins de quarante ans, le taux d'urbanisation qui était de $18 \%$ en 1978 est passé à 56,1 \% en 2015, une progression pour l'essentiel liée aux très importantes migrations des populations rurales chinoises vers les villes. Ce taux de $56,1 \%$ englobe les travailleurs migrants possédant un permis de résidence en zone rurale qui vivent en ville depuis plus de six mois². Les statistiques considèrent ces derniers comme des résidents permanents alors qu'ils ne bénéficient pas des services publics élémentaires offerts aux populations des villes disposant d'un permis de résidence urbain. En réalité, il faut compter qu'environ $20 \%$ des personnes ne possèdent pas de permis de résidence urbain, ce qui ramène le taux d'urbanisation aux alentours de $36,6 \%$. Or ces $20 \%$ représentent des centaines de millions de ruraux migrants qui assurent une part importante du développement des villes chinoises. 


\section{Dette affective et absence de prise en charge sociale}

Ces dernières années, malgré un certain assouplissement, le système de permis de résidence est toujours source de disparité entre les villes et la campagne, de restriction de la mobilité des populations rurales et de leur accès aux services publics dans les villes. En effet, alors que les travailleurs ruraux migrants ont contribué à bâtir "l'usine du monde" qu'est devenue la Chine, ils font face à un manque de reconnaissance : ceux qui ne possèdent pas de permis de résidence urbain ne bénéficient pas des services publics élémentaires, ceux relatifs à l'éducation, la santé, l'emploi ou le logement. Cette situation de "non-intégration" ou de "semi-intégration" a fait d'eux des citoyens urbains de seconde zone. Les grandes migrations de ces familles "éclatées" sont devenues inévitables. Les jeunes travailleurs qui émigrent dans les villes en pleine force de l'âge pour gagner leur vie seront forcés, tels des oiseaux migrateurs, de rentrer terminer leur vie au village.

La plupart des 270 millions de personnes qui composent la population migrante chinoise sont de jeunes travailleurs. Certains emmènent avec eux leurs enfants pour les scolariser en ville, d'autres pas. Cela est à l'origine de deux problèmes : celui des "enfants migrants" et celui des "enfants restés au village”. En 2015, pour la classe d'âge des 0-17 ans, on comptait 36 millions d'enfants migrants (13\% de l'ensemble des enfants) et 61 millions d'enfants restés au village (22\% de l'ensemble des enfants) ${ }^{3}$. Pour ce qui est des enfants en âge de scolarisation obligatoire (6-15 ans), les chiffres officiels publiés en ligne par le ministère de l'Éducation en $2014^{4}$ indiquent que parmi les 138 millions d'enfants en âge de scolarité obligatoire en Chine, 13 mil- lions sont des "enfants migrants" ayant suivi leurs parents en ville et 20 millions sont des "enfants restés au village" n'ayant pas suivi leurs parents, soit près d'un quart de l'ensemble des effectifs.

Ces enfants forment un groupe très important étant donné l'ampleur de l'exode rural en Chine en moins de quarante ans. Le taux de croissance du PNB de la Chine est l'un des plus élevés du monde. Cependant, ce rapide développement économique de la Chine ne doit pas mener à minimiser l'importance de la dette affective engendrée par ces migrations et l'absence de prise en charge de presque cent millions d'enfants migrants ou restés au village au cours des quatre décennies. Dans le cadre
Le CIFA regroupe plusieurs commerces d'import-export. Aubervilliers, avril 2016. (c) Camille Millerand

des politiques en vigueur, ces enfants ne peuvent pas jouir des mêmes opportunités éducatives que les enfants citadins du même âge. Dans les campagnes, les enfants restés au village sont souvent marginalisés. Dispersés dans des villages de plus en plus désertés en raison de la masse de parents partis travailler dans les grands centres urbains, ils 
sont soit élevés par l'un des deux parents resté avec eux ou par des grands-parents âgés, illettrés et parfois en mauvaise santé. Ils sont plus de 9 millions à ne jamais voir leurs parents plus d'une fois par an. Deux millions d'enfants vivraient même seuls. Ces dernières années, l'opinion publique chinoise a été régulièrement bouleversée par des faits divers tragiques survenus à cause de cette situation : des enfants vagabonds retrouvés morts, des suicides collectifs d'enfants ${ }^{5}$, des meurtres d'enseignants par des enfants restés au village, des agressions sexuelles sur fillettes, des formations de bandes d'enfants-voyous. Tout cela a initié une réflexion collective sur la situation de ces presque 100 millions d'enfants ${ }^{6}$. Cependant, en raison de la progression très rapide de l'économie chinoise, il est à craindre que ce problème sociétal subsiste encore de longues années.

\section{Quand enfants migrants et enfants restés au village se retrouvent dans des villes moyennes}

La première génération de travailleurs migrants a laissé place à une seconde génération confrontée à de nouvelles mesures restrictives, mais aussi à de nouvelles opportunités concernant la scolarisation de leurs enfants. D'une part, dans les très grandes villes, le durcissement des mesures de maîtrise des flux migratoires s'est traduit par une restriction des places dans le secteur éducatif public et une difficulté à se maintenir pour les établissements privés dédiés aux enfants migrants. D'autre part, l'assouplissement du système de permis de résidence dans les villes de petite et moyenne importance $e^{7}$ et les réajustements structurels des écoles rurales ont eu pour conséquence de renforcer le désir de la nouvelle génération de travailleurs migrants plus aisée que la génération précédente et attachant plus d'importance à l'éducation - d'envoyer leurs enfants étudier dans les villes proches de leurs villages d'origine. Ainsi est apparue une nouvelle situation où des enfants restés au village et des enfants migrants se retrouvent progressivement dans des villes situées à mi-chemin entre les villages d'origine et les grands centres urbains. Les enfants migrants scolarisés dans les mégapoles repartent étudier dans des agglomérations plus modestes, tandis que les enfants restés au village partent pour de plus grandes agglomérations. Le recul des effectifs dans les établissements ruraux et dans les établissements des grandes villes, les situations de sureffectifs dans les établissements des villes de petite et moyenne importance sont des tendances qui risquent de s'inscrire dans le long terme en Chine.

Les migrations internes ont eu pour effet d'augmenter très rapidement la proportion d'enfants en âge de scolarisation qui sont scolarisés dans les zones urbaines : la proportion est passée de 54,04\% de l'ensemble des enfants en 2009 à 72,55 \% en 2014, soit un gain de 21,5 points en cinq ans. Ce taux d'enfants scolarisés en ville dépasse de loin le taux des résidents permanents (avec ou sans permis de résidence urbain) dans les villes au cours de la même période (46,6 \% en 2009 et 54,8 \% en 2014) ${ }^{8}$. L'augmentation rapide du taux d'enfants scolarisés dans les villes a stimulé tant l'accroissement du taux des résidents permanents que celui des détenteurs de permis temporaires de résidence urbain, ce qui a généré deux conséquences. D’une part, cela a 
entraîné la concentration des ressources éducatives dans les villes de petite et moyenne importance, ce qui a d'ailleurs parfois nourri le développement de nouveaux quartiers périphériques dans ces villes et une offre de logements et de services pour répondre aux besoins des élèves. D'autre part, l'éducation a modifié le rapport traditionnel que les travailleurs venus des campagnes entretenaient avec les terres familiales. À présent, une frange importante de la société est passée d'un mode de vie rural à un mode de vie citadin et souhaite que ses descendants n'aient plus besoin, grâce à léducation, de dépendre du travail de la terre.

\section{Les disparités de la qualité de l'enseignement entre ville et campagne}

Au-delà de l'accroissement rapide du taux d'enfants scolarisés en zone urbaine, trois conclusions s'imposent quant à la répartition structurelle de la population et la nature de ses choix en matière d'éducation. Il est d'abord malheureusement très probable que l'intégration des nouveaux flux de migrants ruraux perdure sur un mode d'"intégration urbaine partielle". Les enfants migrants peineront à avoir accès à une éducation publique de qualité dans les grandes villes et les parents feront sûrement souvent le choix d'une scolarisation dans les campagnes dont ils sont issus ou, quand les parents ont plus de moyens, dans des villes secondaires, en périphérie des grandes métropoles, où les ressources éducatives sont meilleures. Ensuite, dans les villes moyennes et les chefs-lieux de district, l'essor urbain rapide (dû à la construction de logements, à la transformation de vieux quartiers en villes nouvelles et àla mise en place de zones de développement économique) a permis un relatif accroissement des ressources dédiées au secteur éducatif public. Enfin, l'urbanisation de petites villes et de villages génère une centralisation des ressources éducatives en sup- primant nombre d'écoles dispersées dans les campagnes. Comme le centre de gravité des établissements se déplace à un rythme rapide des villages vers les villes, cela oblige la population en âge scolaire à suivre le mouvement et à partir s'instruire dans des villes plus importantes.

À ces trois facteurs s'ajoute la nette disparité de la qualité de l'enseignement dispensé entre villes et campagne. Les familles rurales (non migrantes) font aussi des choix éducatifs différents. Si les familles un peu plus aisées que la moyenne envoient leurs enfants faire leurs études en ville, les familles modestes n'ont pas vraiment d'autre choix que celui des établissements ruraux de moindre qualité proches de chez eux. Les enfants des premières viennent grossir les rangs des enfants migrants en âge de scolarisation afin de profiter du niveau scolaire plus élevé en ville. Les chiffres de 2014 révèlent que le taux de scolarisation en ville pour les élèves en primaire a atteint $67,73 \%$,
Les enfants migrants peineront à avoir accès à une éducation publique de qualité dans les grandes villes et les parents feront souvent le choix d'une scolarisation dans les campagnes dont ils sont issus ou, quand les parents ont plus de moyens, dans des villes secondaires, en périphérie des grandes métropoles, où les ressources éducatives sont meilleures. tandis que celui des élèves au collège a atteint 82,93 \% de l'ensemble des enfants scolarisés 9 . Bien qu’après 2012, les petites écoles rurales ainsi que les écoles primaires et les collèges des chefs-lieux de district aient peu à peu repris de la vigueur, leur fermeture à grande échelle ainsi que la rigidité du "règlement de la gestion de la sécurité du transport scolaire" imposent souvent à ces enfants issus des familles rurales modestes de vivre en internat pour pallier leur éloignement. Le taux d'élèves internes est respectivement passé entre 2009 et 2014 de 11,67 \% à 16,25 \% en primaire et de 52,26 \% à 64,83 \% au collège ${ }^{10}$. Que les familles rurales modestes soient privées du droit de s'exprimer et d'intervenir sur ce sujet reste un problème encore plus épineux que les seules condi- 
tions matérielles. En bref, le recul des établissements ruraux et la saturation des établissements urbains sont un phénomène difficile à gérer dans une Chine qui s'urbanise à un rythme soutenu.

\section{La reproduction des inégalités sociales dans les établissements scolaires}

La mobilité des travailleurs ruraux engendre des problématiques éducatives radicalement différentes pour les établissements scolaires ruraux et urbains. Dans les zones rurales, la diminution continue des effectifs d'enfants en âge de scolarisation a entraîné une réduction progressive de la taille des établissements scolaires. Depuis 2001, l'orientation politique au niveau national est celle de la concentration des écoles au sein des centres urbains dans un souci d'optimisation des ressources et d'amélioration de la qualité de l'enseignement. En l'espace d'une décennie, de nombreux établissements scolaires dispersés dans des campagnes reculées ont ainsi été fermés par décision des autorités administratives pour être transférés vers les centres urbains. Malgré tout, en vertu du principe selon lequel la scolarité doit s'effectuer au plus près de son domicile, une partie des établissements ruraux ont été maintenus, mais ils continuent de faire face au manque d'effectifs. Inversement, dans les villes, les effectifs d'élèves augmentent à un rythme soutenu et la pression sur les établissements ne cesse de s'accroître. Dans les villes, un double défi a émergé : celui des très grands établissements et celui des classes surchargées. Bien que les autorités aient adopté toutes sortes de mesures dans l'espoir de maîtriser la capacité d'accueil des établissements en zone urbaine (telles que la construction de nouveaux établissements, la répartition et la restriction des effectifs), les établissements continuent à faire face à de multiples et complexes problèmes de gestion. Dans les villes, notamment dans les très grandes, où certains établissements sont confron- tés à l'arrivée ininterrompue d'enfants migrants, les chefs d'établissement adoptent souvent une politique de création de "classes spécifiques" pour séparer les élèves des familles urbaines de ceux issus de familles migrantes ${ }^{11}$. Les arguments invoqués sont d'éviter la survenue de conflits entre les élèves urbains et ruraux, mais aussi de faciliter l'enseignement et la gestion au quotidien. Cette pratique de création de "classes spécifiques" induit chez l'élève migrant un processus inconscient d'identification à une classe sociale au sein de l'établissement avant même son intégration à la vie active. Ainsi, tandis que les établissements scolaires des villes intensifient leur fonction d"ascension sociale individuelle" par des moyens sélectifs, ils encouragent aussi le cycle de la "reproduction sociale". Dans les établissements situés en zone rurale, cette stratégie de création de "classes spécifiques" qui s'apparente à une forme de ségrégation sociale n'a bien entendu aucun sens. La diminution de la population en âge d'être scolarisée à la campagne a conduit un grand nombre d'écoles à constituer des classes uniques à effectifs réduits. Mais le désagrément de ces classes uniques a fait émerger une autre problématique de gestion de classe : les "classes à emplacement imposé". Si les "classes spécifiques" sont le mécanisme principal de reproduction des inégalités sociales au sein des grands établissements scolaires dans les villes, les "classes à emplacement imposé" sont, quant à elles, le mécanisme principal par lequel s'opère la reproduction des inégalités sociales au sein des petites structures scolaires en zone rurale.

\section{De la géographie de la classe à l'imposition d'une place sociale}

Plusieurs enquêtes menées sur le terrain dans quelques établissements du centre de la Chine ${ }^{12}$ ont permis de découvrir qu'au sein des plus petites structures scolaires essentiellement composées d'enfants de migrants restés au village, le fait d'avoir 
des classes uniques a conduit les enseignants à opérer avec des classes à "emplacement imposé". Il s'agit autant de maintenir le calme parmi les élèves que d'offrir à tous la possibilité de réussir l'examen d'entrée dans des lycées généraux considérés comme offrant un avenir prometteur, et d'échapper ainsi aux lycées professionnels qui offrent un avenir moins engageant.

Si l'on prend l'exemple d'une école rurale essentiellement composée d'enfants de migrants, située dans une zone reculée de la Chine, les élèves des petites classes sont placés selon une règle que les responsables éducatifs considèrent comme juste, à savoir celle de la séparation entre les bons et les mauvais éléments, laquelle s'opère sur le modèle : "bons au milieu, moins bons sur les côtés". En fait, sur les quatre rangées que comporte la classe, il s'agit de placer dans les deux rangées centrales les élèves dont le comportement et les résultats sont jugés excellents et de disposer sur les deux rangées extérieures ceux au comportement et aux résultats médiocres. Les "bons éléments" du milieu sont censés venir en aide à leurs camarades "faibles" situés de chaque côté en formant ainsi des binômes d'entraide. Chaque semaine les élèves changent de place, mais le changement de place ne s'opère que d'un point de vue vertical et jamais horizontal.

Dans le secondaire, les élèves sont placés en fonction de leur capacité à réussir les examens et de leurs choix professionnels. Désormais, c'est une stratégie de placement horizontal qui l'emporte. Le dernier rang est occupé par des élèves sans motivation ni potentiel et qui n'ont aucun espoir d'être admis dans un lycée général ou professionnel. Les deuxième et troisième rangs en partant du fond sont occupés par des élèves pour qui intégrer un lycée à enseignement général est hors de porté et qui, constamment en risque de décrochage scolaire, attendent la fin du collège pour s'orienter vers la filière professionnelle. Au premier rang se trouvent des élèves d'un niveau honorable qui ont le potentiel pour intégrer un lycée rural où l'enseignement est de qualité moyenne. Ces élèves qui sont considérés comme disposant d'un faible esprit d'initiative sont sans cesse sous la surveillance et l'attention de leurs enseignants. Enfin, les deuxième et troisième rangs sont occupés par des élèves dont les résultats sont supérieurs à la moyenne ou qui sont de bons éléments, élèves qui auront la possibilité d'entrer dans un lycée général de district ou de petite ville proche.

Derrière la nature "fonctionnelle" ou "significative" de ces agencements de classe et dispositions d'élèves se profile en réalité une stratégie qui ne dit pas son nom et qui fait que la plupart des enfants restés au village s'identifient à leur position en classe comme à une classe sociale (central-marginal ; bon-mauvais), laquelle rejoint tant la nature sélective des normes éducatives chinoises que la logique de reproduction de classe au sein de la société. L'assignation à une place donnée dans la classe est un facteur-clé qui accélère ce processus d'appartenance sociale au cours de la socialisation individuelle. Les élèves d'établissements en zone rurale qui sont pour beaucoup sans leurs parents ne peuvent qu'anticiper la place qu'ils occuperont dans la société en fonction de la place qu'ils occupent dans la classe. Après le collège, nombre de ces élèves éliminés par le système très sélectif des examens deviendront, à l'instar de leurs parents, la nouvelle génération des travailleurs qui ira grossir les rangs de la main-d'œuvre de "l'usine du monde".

Texte traduit du mandarin par Stéphane Lévêque. 